\title{
Der Nürnberger Trichter
}

\section{Erhard Taverna}

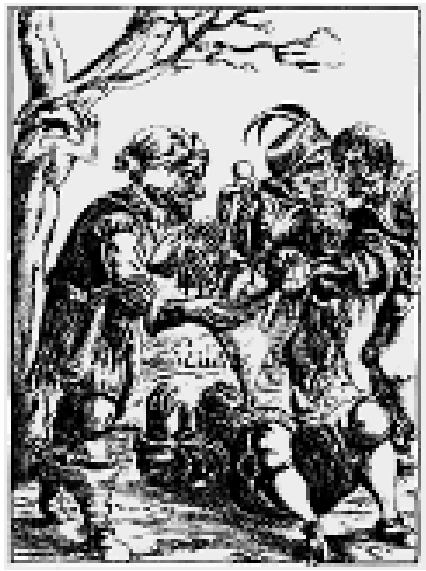

Der Weisheitstrichter wird in einem Rechenbuch erstmals erwähnt: «... so einer die Kunst ihm könnte eingiessen.» Oder eintrichtern, wie die drei Männer auf einem Kupferstich des 17. Jahrhunderts; eine passiv-gewaltsame Lehr- und Lernmethode, die sich bisher nie ganz durchgesetzt hat. Lernen bleibt anstrengend, auch wenn die Plackerei akkreditiert, praxisrelevant und interaktiv in vielen Verpackungen vorliegt. Das Informationsverhalten niedergelassener Ärzte in der Schweiz ist Thema einer Dissertation an der Universität St. Gallen [1]. Darin werden die Informationsmedien unterschieden nach: personenbezogenen, wie Schulungsveranstaltungen und Kongresse, nach unpersönlich-klassischen, wie Fachartikel und Broschüren, und nach interaktiv-neuen, wie Internetportale, elektronische Datenträger und Mobile Services.

Gemäss dieser Studie steht fest, dass der persönliche Fachaustausch mit Kollegen die wichtigste Informationsquelle darstellt, und es nach Meinung der Befragten auch bleiben wird. Online-Informationen werden zwar relativ wenig genutzt, gelten aber als qualitativ gutbewertetes Medium der Zukunft. Angebote, die sich an den ehemals klassischen Printmedien orientieren, werden am meisten aufgesucht, sehr viel seltener die passwortgeschützten, artspezifischen Internetseiten.

Ein gutes Beispiel für die letztere, passwortgeschützte Art ist www.univadis.ch - Das Portal zum Wissen. Eine Redaktion von fünf Personen ist für den vielseitigen Inhalt verantwortlich: Marcel Müller, Internetexperte und Germanistikstudent in Zürich, Dr. med. Ueli Kappeler, Chirurgieassistent am Kantonsspital Aarau, Dr. med. Jean-Gabriel Jeannot, Praktiker in St-Blaise und Herausgeber einer eigenen Plattform www. medixx.ch, und Prof. Klaus Neftel, medizinischer Chefarzt am Berner Zieglerspital von 1989 bis 2000 und Gründer eines Fortbildungscurriculums, das Fortbildungsnachmittage mit Internetmodulen verbindet. Klaus Neftel betreut auch

ein weiteres Portal www.tellmed.ch, wo ebenfalls SGIM-akkreditierte Kurse angeboten werden.

Es gibt inzwischen zahlreiche Portale mit unterschiedlichen Sponsoren, die nach der Methode vom Lernmodul zum Quiz, zum Test und bei Bestehen zum ausgedruckten Zertifikat, Lektionen und Kurse anbieten. Was www. univadis.ch darüber hinaus auszeichnet, ist eine umfangreiche Bibliothek mit zahlreichen Volltextbüchern, Zugang zu renommierten Nachschlagewerken und ein umfangreiches Bildarchiv. Bemerkenswert sind die Websiterezensionen, die durch den redaktionellen Vorfilter eine Menge Suche ersparen und nur das Interessanteste zusammenfassen. Zurzeit ein Lernprogramm zur Wundversorgung der Universität Bern, einen interaktiven Anatomieatlas der Universität Washington oder den Simulator für ärztliche Konsultationen vom Genfer Arzt Marc-André Raetzo. Klaus Neftels pädagogisches Flair und literarische Fähigkeit sorgen für eine ausgezeichnete wöchentliche Kolumne rund um das Gesundheitswesen mit selbstverfassten und ausgewählten Artikeln. Sein Team hat ein Portal von hoher Qualität erarbeitet, das auch andere Elemente wie Praxistools, Datenbanken, hilfreiche Links, Kongresskalender, Verzeichnisse, medizinische Suchmaschinen und weitere OnlineDienste einbezieht.

Die praktische Medizin ist weder eine Wissenschaft noch eine Kunst. Hausärzte, Spezialisten und Spitalärzte sind Handwerker, die den widersprüchlichsten Anforderungen zu genügen haben. Die Kunst besteht wohl darin, im Dschungel der Informationen vom rechten Weg nicht abzukommen. Eintrichtern ist dabei das eine, verdauen aber das wichtigere. Was als Resultat davon auf dem eigenen Fachgebiet Erfolg hat oder als Kompetenz wahrgenommen wird, hat viele Wurzeln. Charaktereigenschaften wie Empathie, Intelligenz, Selbsterkenntnis und Ausdauer entscheiden unter anderem über Wissen und Fähigkeiten. 